\title{
AN EXPERIMENTAL STUDY OF THE BENDING BEHAVIOR OF CALL HYBRID COMPOSITES
}

\author{
T. X. Mao, J. H. Han \\ Institute of Mechanics, Academia Sinica, 15 Zhong Guan Cun Road, Beijing, People's Republic of China \\ X. L. Qing \& F. L. Dai \\ Department of Engineering Mechanics, Qinghua University, Beijing, People's Republic of China
}

(Received 24 January 1994; revised version received 1 August 1994; accepted 22 November 1994)

\begin{abstract}
The bending behavior and damage characteristics of CALL (Carbon fiber/epoxy/AL Laminate) hybrid composites have been studied by moiré interferometry. The shear strain distribution along the cross-section and the forms of damage of bending beams are obtained. The results show that the magnitude of the shear strain in a carbon/epoxy layer is obviously larger than that in a corresponding aluminum layer and the shear strain distribution of a CFRP layer along the cross-section conforms basically to a parabolic distribution curve, as for the shear strain distribution in aluminum layers along the cross-section. Shear damage, either in the interfaces or in carbonfiber/epoxy laminae, and tensile failure of CFRP laminae in the tension surface represent, respectively, the damage forms of the longitudinal and transverse bending specimen.
\end{abstract}

Keywords: CALL, moiré interferometry, bending, shear strain

\section{INTRODUCTION}

A series of research studies on an aramid-fiberreinforced aluminum alloy laminate (ARALL) have been carried out in The Netherlands. The material is built up as a laminated sheet material with thin high-strength aluminum alloy sheets and strong unidircctional or woven aramid or glass fibers impregnated with a thermoset or thermo-plastic adhesive. It was reported that ARALL possessed excellent fatigue properties, and had a tensile strength as high as that of the composite with the impact resistance and machinability of the metal.

Based on the outstanding properties of carbonfiber-reinforced plastics (CFRP), Carbon/epoxy/ ALuminum Laminate (CALL) was valued for its low specific gravity, low thermal expansion, high strength and long fatigue life. ${ }^{1,2}$
Moiré interferometry is a modern optical/ mechanical measurement method developed in the 1980s. ${ }^{3,4}$ Possessing the advantages of high sensitivity, high quality of fringe patterns, in situ measurement and whole-field analysis, it has been widely used in the experimental studies on new materials. In this paper, its use in investigating the bending behavior and failure characteristics of unidirectional reinforced CALL hybrid composite in the longitudinal and transverse directions is discussed.

\section{SPECIMENS AND EXPERIMENTAL SET-UP}

The unidirectional CALL is composed of 13 layers of surface-treated aluminum alloy sheet and 12 layers of carbon/epoxy composite, and is prepared by hot pressing. The carbon/epoxy layer is composed of two prepregs with a thickness of $0.125 \mathrm{~mm}$ and the aluminum sheet is $0.2 \mathrm{~mm}$ thick. The longitudinal and transverse specimens were cut in the fiber direction and perpendicular to the fiber direction, respectively (Fig. 1).

A high-frequency crossed-line grating is replicated onto the surface of the specimen and it deforms together with the specimen when loads are applied. The resulting fringe patterns correspond to those of the moire type with $2400 \mathrm{lmm}^{-1}$. In-plane $U$ and $V$ displacement fields (in the respective $x$ and $y$ directions) are determined, with sensitivities of $0.417 \mu \mathrm{m}$ per fringe order.

The experimental set-up is shown in Fig. 2. M1, M2 and $\mathrm{M} 3$ are three adjustable mirrors. We let beams of collimated light from sections $\mathrm{C}$ and $\mathrm{D}$ be blocked by an opaque screen and the beams from $A$ and $B$ reach the apparatus. After one part is reflected from the mirror M1, two beams reach the specimen and intersect at an angle of $2 \alpha$ in the horizontal plane to form a virtual reference grating with lines perpendicular to the $x$ direction. Next, we let sections $\mathrm{A}$ and $\mathrm{B}$ of 


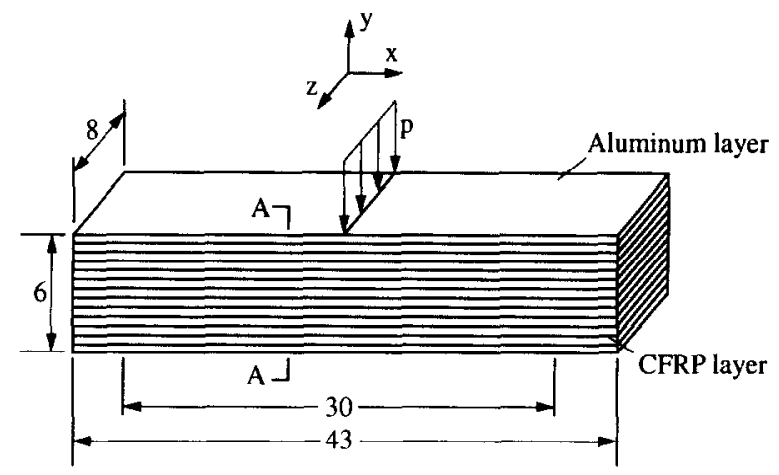

Fig. 1. Specimen characteristics (dimensions in $\mathrm{mm}$ ).

the incoming beams be blocked, and beams from $\mathrm{C}$ and $\mathrm{D}$ reach the specimen after being reflected from the mirrors $\mathrm{M} 2$ and $\mathrm{M} 3$ respectively. They intersect at an angle of $2 \alpha$ in the vertical plane and form a virtual reference grating with lines perpendicular to the $y$ direction. The frequency of each virtual reference grating is $f=(2 \sin \alpha) / \lambda$, where $\lambda$ is the wavelength of the light. Each reference grating interacts with the similarly oriented lines of the specimen grating to form the interference patterns recorded by the camera. The patterns are contour maps of displacement governed by the relationships:

$$
U=\frac{1}{f} N_{x}, \quad V=\frac{1}{f} N_{y}
$$

where $U$ and $V$ are in-plane $x$ and $y$ components of displacement at any point; $N_{x}$ and $N_{y}$ are the fringe orders at the point in the corresponding fringe patterns, i.e. the patterns formed by grating lines perpendicular to the $x$ and $y$ directions respectively. In this work, $f$ was $24001 \mathrm{~mm}^{-1}$. A He-Ne laser power source was used at a wavelength of $0.6328 \mu \mathrm{m}$ and a power of $50 \mathrm{~mW}$.

\section{EXPERIMENTAL RESULTS AND ANALYSIS}

\section{In-plane displacement and shear strain}

Representative moiré interferometry fringe patterns are shown in Fig. 3(a) and (b) and Fig. 4(a) and (b) for two different load levels for longitudinal and transverse specimens, respectively. The in-plane shear strain can be obtained from fringe patterns of the $U$ and $V$ fields according to the following formula:

$$
\gamma_{x y}=\frac{\partial U}{\partial y}+\frac{\partial V}{\partial x}=\frac{1}{f}\left(\frac{\partial N_{x}}{\partial y}+\frac{\partial N_{y}}{\partial x}\right)
$$

where $f=24001 \mathrm{~mm}^{-1}$ is the frequency of the virtual reference grating; $N_{x}$ and $N_{y}$ are the fringe orders in the corresponding fringe patterns for $U$ and $V$ displacement field.

The shear strain distribution curve across AA section are shown in Fig. 5(a) and (b) for longitudinal specimens at a load level of $200 \mathrm{~kg}$ and for a

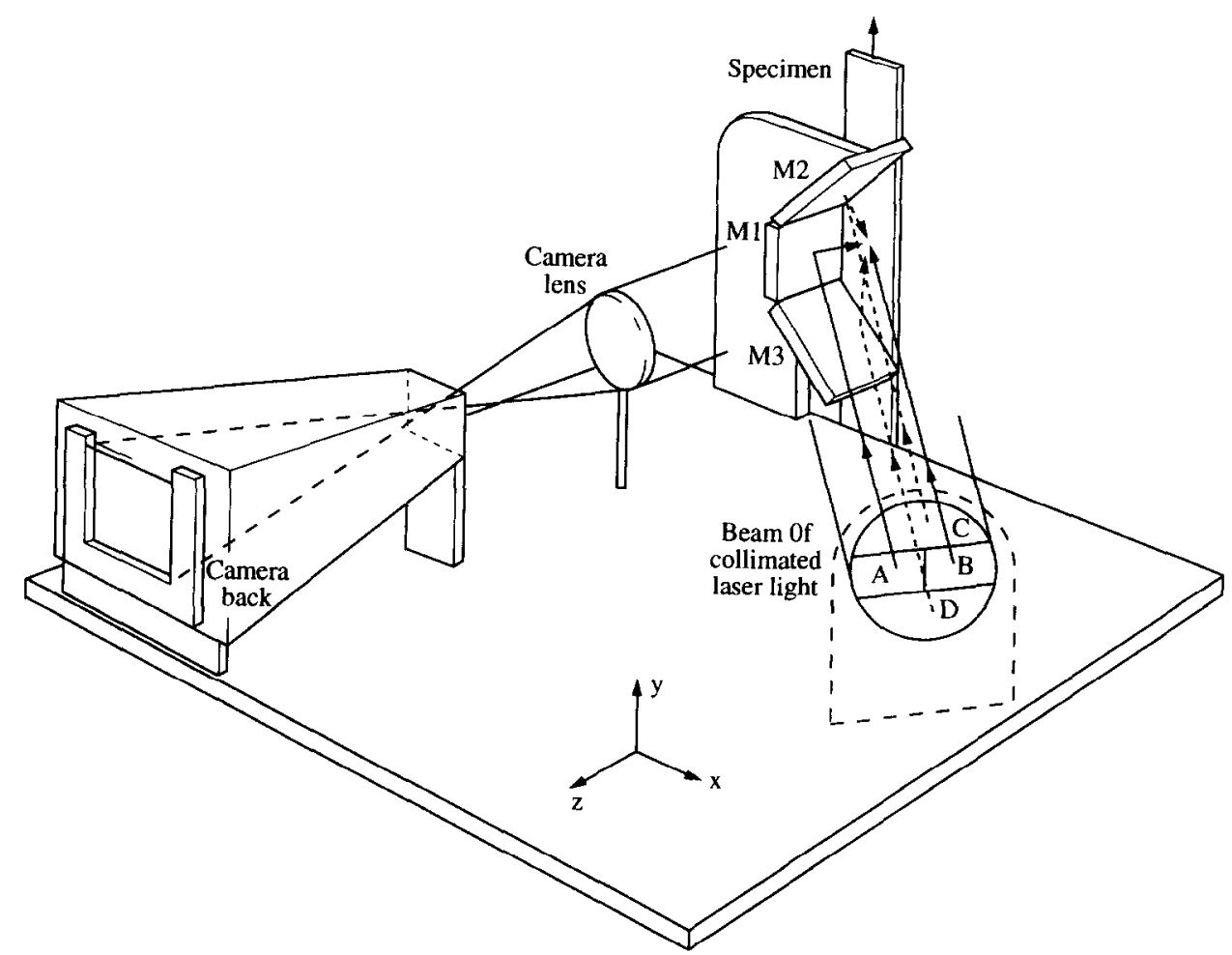

Fig. 2. Four-beam optical arrangement to produce the $N_{x}$ pattern with beams $\mathrm{A}$ and B and the $N_{y}$ pattern with beams $\mathrm{C}$ and $\mathrm{D}$. 


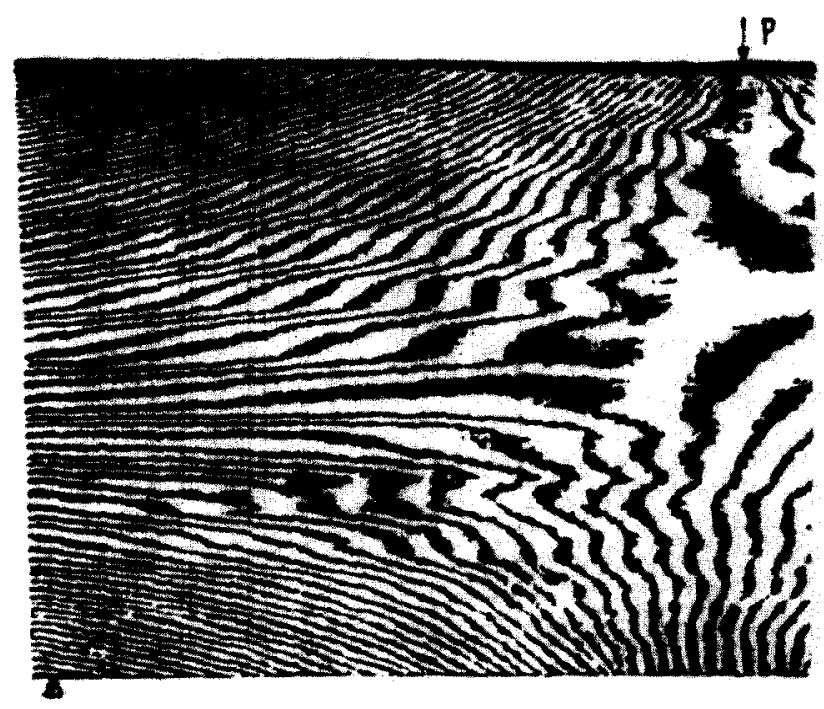

(a)

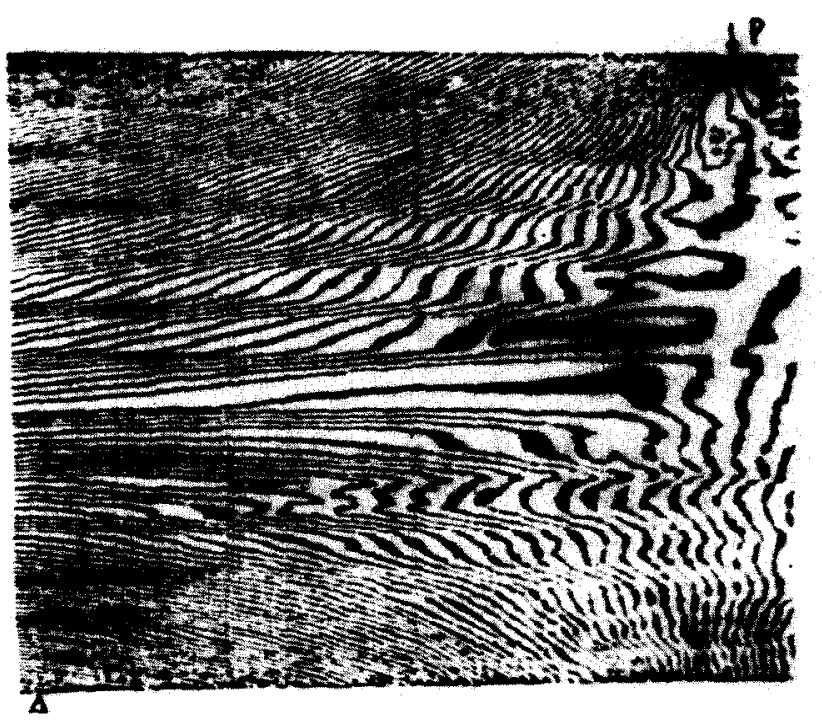

(b)

Fig. 3. $U$ displacement field for longitudinal specimen. (a) $P=125 \mathrm{~kg}$, (b) $P=200 \mathrm{~kg}$.

transverse specimen at a load of $58 \cdot 3 \mathrm{~kg}$, respectively. It is obvious from Fig. 5 that no matter whether for a longitudinal or a transverse specimen, the shear strains in the CFRP layers are larger than those in aluminum layers, but the envelopes of these strains are nearly parabolic and similar to those that would be obtained for an isotropic specimen. The reason for the difference of shear strain in the two kinds of layers is the difference in the moduli of the two kinds of material. The shear modulus is $27.7 \mathrm{GPa}$ for aluminum, $7 \mathrm{GPa}$ for the in-plane shear modulus, $G_{12}$ of the unidirectional CFRP lamina and $3 \mathrm{GPa}$ for the epoxy matrix. The shear behavior of the CFRP layers is determined by the shear moduli, $G_{12}$ and $G_{23}$, for the longitudinal and transverse specimens, respectively. The relationship between these and $G_{13}$ and the

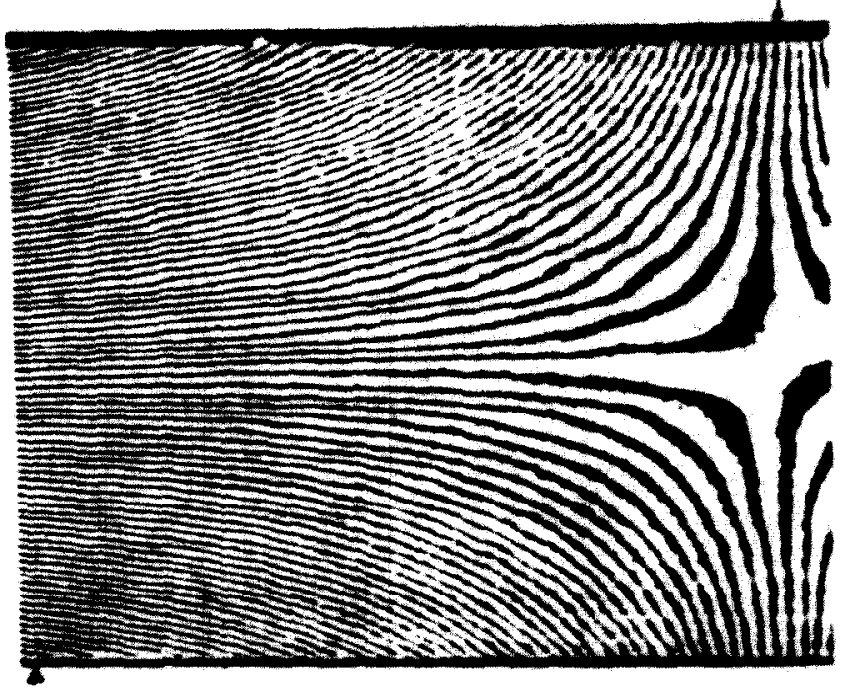

(a)

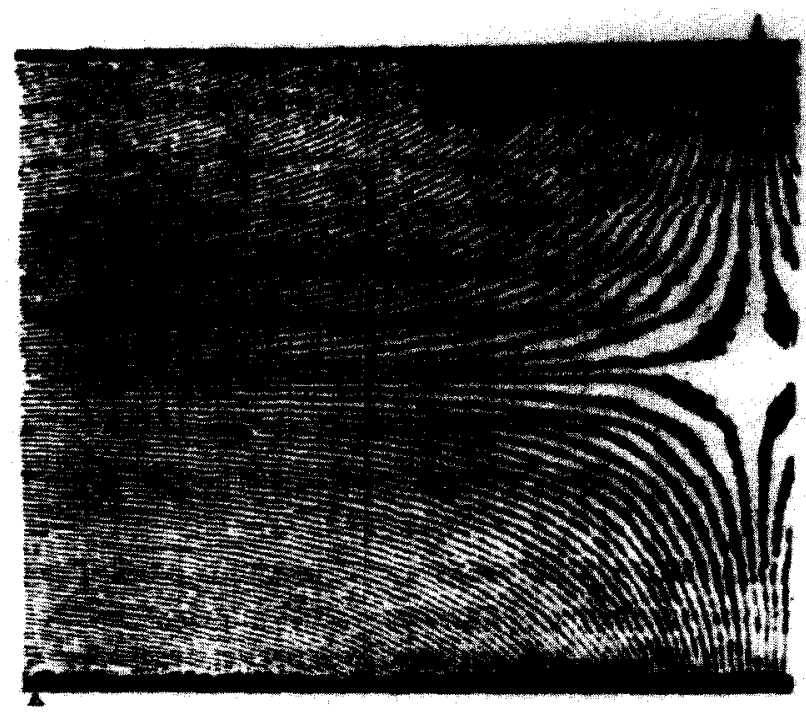

(b)

Fig. 4. $U$ displacement field for transverse specimen. (a) $P=41.6 \mathrm{~kg}$, (b) $P=58.3 \mathrm{~kg}$.

shear modulus of epoxy matrix, $G^{\prime}$, satisfies the following formula:

$$
G_{12}>G_{13}>G_{23}>G^{\prime}
$$

Therefore the shear moduli of the CFRP layers, no matter whether for a longitudinal or a transverse specimen, are between $3 \mathrm{GPa}$ and $7 \mathrm{GPA}$, which means they are only one fourth to one ninth of the aluminum modulus. As shown in Fig. 5, for a longitudinal specimen the maximum shear strains are $8600 \mu \varepsilon$ and $3000 \mu \varepsilon$ in the CFRP layer and the aluminum layer, respectively. The equivalent strains are $3600 \mu \varepsilon$ and $1700 \mu \varepsilon$, respectively, for transverse specimen.

\section{Damage characteristics}

The failure loads are $267 \mathrm{~kg}$ and $66 \mathrm{~kg}$ for longitudinal and transverse specimens, respectively. The failure 


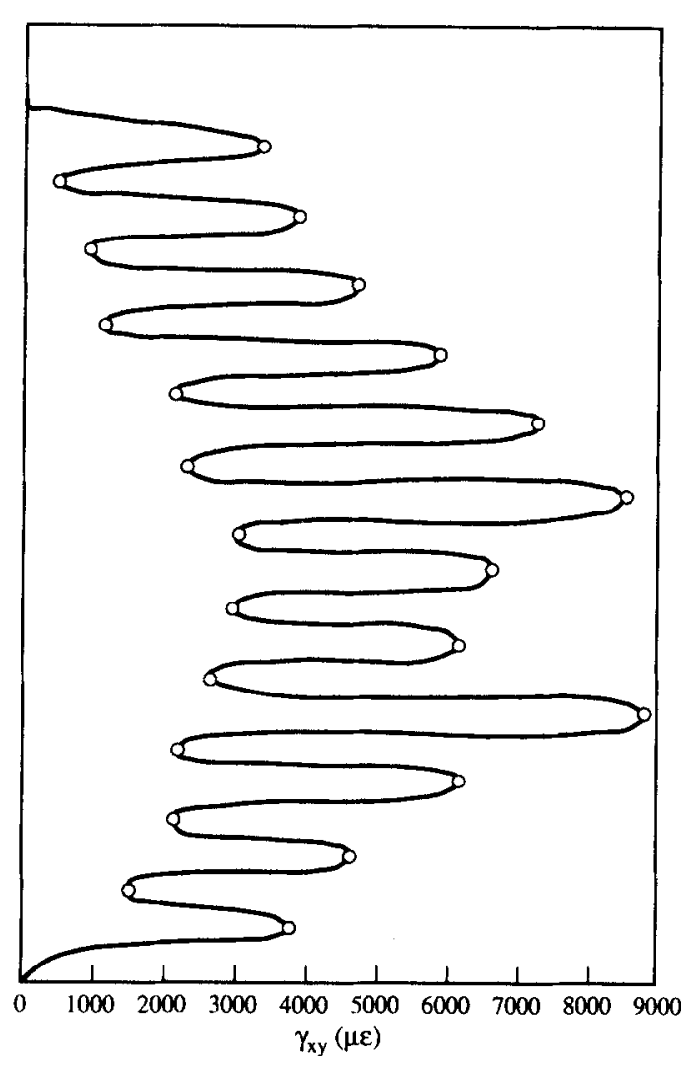

(a)

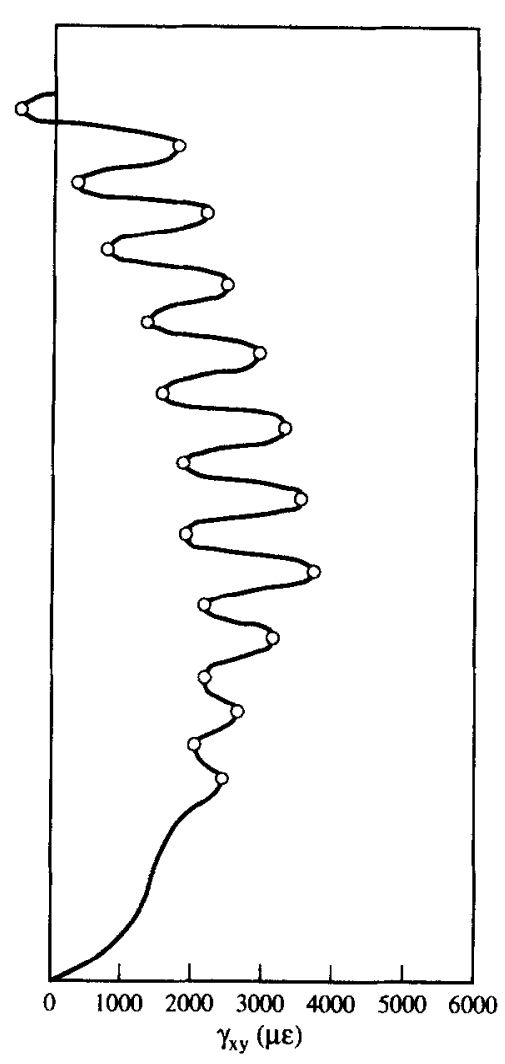

(b)

Fig. 5. Shear strain distribution along AA sections. (a) Longitudinal specimen, (b) transverse specimen.

forms are quite different for these two kinds of specimen.

(a) The main forms of bending failure for longitudinal specimens are delamination and the localization of shear damage caused by the matrix failure in the CFRP layers. The specimen does not completely lose its load-carrying capacity after delamination or shear damage has appeared, but the damage will expand with increase in load until it extends through the whole specimen, leading to the final failure.

(b) Tensile failure of the CFRP layer on the tensile surface is the main form of bending failure for a transverse specimen. Tensile cracks in the $90^{\circ}$ CFRP layer localize the damage. The damage also causes necking, at the damage site, in the aluminum layer adjacent to the damaged CFRP layers, which then leads to the final failure of the specimen. It was found in these tests that the crack appeared at a load of $66 \mathrm{~kg}$, but the final failure did not come until the load reached $70 \mathrm{~kg}$. After the test, a groove was found along the width direction on the tensile surface of the beam. This is the result of necking in the aluminum layer. The maximum tensile strain and the maximum shear strain in longitudinal specimens at loads of $200 \mathrm{~kg}$ and for transverse specimens at loads of $58.3 \mathrm{~kg}$ obtained from moire fringe patterns of the displacement field are shown in Table 1.
Table 1. Maximum tensile and shear strain from moiré fringe patterns

\begin{tabular}{lcc}
\hline \multicolumn{1}{c}{ Type of specimen } & $\begin{array}{c}\text { Long- } \\
\text { itudinal }\end{array}$ & $\begin{array}{c}\text { Trans- } \\
\text { verse }\end{array}$ \\
\hline Load (kg) & 200 & $58 \cdot 3$ \\
Max. tensile (aluminum layer) & $4746 \mu \varepsilon$ & $6130 \mu \varepsilon$ \\
Strain (CFRP layer) & $4370 \mu \varepsilon$ & $5880 \mu \varepsilon$ \\
Max. shear (aluminum layer) & $3000 \mu \varepsilon$ & $1700 \mu \varepsilon$ \\
Strain (CFRP layer) & $8600 \mu \varepsilon$ & $3600 \mu \varepsilon$ \\
\hline
\end{tabular}

It is found that the value of maximum tensile strain is only half of the maximum shear strain in the CFRP layer in a longitudinal specimen. But the tensile strength in the fiber direction is much larger than the interlaminar shear strength, so it is easy to understand why interlaminar or shear failure are the main forms of damage for this kind of specimen. For the transverse specimen, the maximum tensile strain is larger than the maximum shear strain in the CFRP layer. But the tensile strength in the transverse direction of the CFRP lamina is much lower, and therefore tensile failure appeared much earlier than interlaminar or shear failure in these specimens.

It is interesting to note that the maximum tensile strain $(5880 \mu \varepsilon)$ in a transverse specimen at a load of $58.3 \mathrm{~kg}$ is even larger than the maximum tensile strain 
$(4370 \mu \varepsilon)$ in a longitudinal specimen at a load of $200 \mathrm{~kg}$.

\section{CONCLUSIONS}

(a) Moire interferometry is a powerful method for the experimental investigation of CALL hybrid composites.

(b) For both longitudinal and transverse specimens, the shear strains in the CFRP layers are obviously greater than those in the aluminum layers, but the envelope of $\gamma_{x y}$ graphs is nearly parabolic as in the case of an isotropic specimen.

(c) The bending failure mode for a longitudinal specimen is shear failure either at the interface or in the carbon/epoxy layers. Failure in a transverse specimen is caused by the tensile failure of the CFRP layers on the tension surface of the beam.

These experimental results provide an important basis for further investigation on CALL hybrid composites.

\section{ACKNOWLEDGEMENTS}

The authors would like to thank Prof. R. J. Wu, Prof. T. R. Hu and Dr K. Sun of the Institute of Composite Materials, Shanghai JiaoTong University for their support and help. We would also like to acknowledge the financial support of the National Natural Foundation of China and the Laboratory of Structure and Vibration, Xinghua University.

\section{REFERENCES}

1. Mao, T. X. \& Han, J. H., Tensile fracture of carbon/epoxy aluminum laminates. Proc. ISCMS/II, Beijing, China, 3-7 August 1992, pp. 899-904.

2. Sun, K., Zhang, X. G., Hu, T. Y. \& Wu, R. J., Possibility of galvanic corrosion in interlayer of CALL. Proc. ISCMS/II, Beijing, China, 3-7 August 1994, pp. 905-10.

3. Post, D., Moiré interferometry for damage analysis of composites. Exp. Mech., July 1983, pp. 17-20.

4. Han, J. H. \& Mao, T. X., The application of moiré interferometry. Acta Mech. Sinica, 7(4) (1991) 376-82. 